\title{
BRookhauren
}

BNL-77545-2007- CP

\section{Touschek lifetime calculations and simulations for NSLS-II}

\author{
C. Montag, J. Bengtsson, B. Nash \\ Presented at the $22^{\text {nd }}$ Particle Accelerator Conference (PAC) \\ Albuquerque, New Mexico \\ June $25-29,2007$ \\ Collider-Accelerator Department \\ Brookhaven National Laboratory \\ P.O. Box 5000 \\ Upton, NY 11973-5000 \\ www.bnl.gov
}

\begin{abstract}
Notice: This manuscript has been authored by employees of Brookhaven Science Associates, LLC under Contract No. DE-AC02-98CH10886 with the U.S. Department of Energy. The publisher by accepting the manuscript for publication acknowledges that the United States Government retains a non-exclusive, paid-up, irrevocable, world-wide license to publish or reproduce the published form of this manuscript, or allow others to do so, for United States Government purposes.
\end{abstract}

This preprint is intended for publication in a journal or proceedings. Since changes may be made before publication, it may not be cited or reproduced without the author's permission. 


\section{DISCLAIMER}

This report was prepared as an account of work sponsored by an agency of the United States Government. Neither the United States Government nor any agency thereof, nor any of their employees, nor any of their contractors, subcontractors, or their employees, makes any warranty, express or implied, or assumes any legal liability or responsibility for the accuracy, completeness, or any third party's use or the results of such use of any information, apparatus, product, or process disclosed, or represents that its use would not infringe privately owned rights. Reference herein to any specific commercial product, process, or service by trade name, trademark, manufacturer, or otherwise, does not necessarily constitute or imply its endorsement, recommendation, or favoring by the United States Government or any agency thereof or its contractors or subcontractors. The views and opinions of authors expressed herein do not necessarily state or reflect those of the United States Government or any agency thereof. 


\title{
TOUSCHEK LIFETIME CALCULATIONS AND SIMULATIONS FOR NSLS-II
}

\author{
C. Montag, J. Bengtsson, B. Nash, Brookhaven National Laboratory, Upton, NY 11973, USA
}

\section{Abstract}

The beam lifetime in most medium energy synchrotron radiation sources is limited by the Touschek effect, which describes the momentum transfer from the transverse into the longitudinal direction due to binary collisions between electrons. While an analytical formula exists to calculate the resulting lifetime, the actual momentum acceptance necessary to perform this calculation can only be determined by tracking. This is especially the case in the presence of small vertical apertures at insertion devices. In this case, nonlinear betatron coupling leads to beam losses at these vertical aperture restrictions. In addition, a realistic model of the storage ring is necessary for calculation of equilibrium beam sizes (particularly in the vertical direction) which are important for a self-consistent lifetime calculation.

\section{INTRODUCTION}

In most modern medium energy $(\approx 3 \mathrm{GeV})$ synchrotron radiation sources, the beam lifetime is limited by the Touschek effect, which describes the collision of two electrons inside a bunch due to transverse focusing. These collisions lead to momentum transfer from the transverse planes into the longitudinal direction. In the event that the resuting particle momentum exceeds the momentum acceptance $\delta_{\mathrm{acc}}$ of the storage ring, the electron is lost.

The resulting Touschek lifetime is calculated as [1]

$$
\tau^{-1}=\frac{r_{e}^{2} c q}{8 \pi e \gamma^{3} \sigma_{s}} \cdot \frac{1}{C} \cdot \oint_{C} \frac{F\left(\left[\frac{\delta_{\mathrm{acc}}(s)}{\gamma_{\sigma_{z^{\prime}}}(s)}\right]^{2}\right)}{\sigma_{x}(s) \sigma_{x^{\prime}}(s) \sigma_{z}(s) \delta_{\mathrm{acc}}^{2}} \mathrm{~d} s
$$

where $r_{e}$ denotes the classical electron radius, $q$ the bunch charge, $\sigma_{s}$ the bunch length, $C$ the circumference of the storage ring, and $\sigma_{x}(s)$ and $\sigma_{z}(s)$ the rms horizontal and vertical beam radii, including the dispersion term. $c$ is the vacuum velocity of light, $e$ the electron charge, and $\gamma$ the relativistic Lorentz factor of the beam.

The beam divergence $\sigma_{x^{\prime}}$ for $x=0$ is expressed as

$$
\sigma_{x^{\prime}}=\frac{\epsilon_{x}}{\sigma_{x}(s)} \sqrt{1+\frac{\mathcal{H}(s) \sigma_{\delta}^{2}}{\epsilon_{x}}}
$$

where $\mathcal{H}(s)$ is the chromatic invariant

$$
\mathcal{H}(s)=\gamma_{x} \eta^{2}+2 \alpha_{x} \eta \eta^{\prime}+\beta_{x} \eta^{2}
$$

and $\epsilon_{x}$ and $\sigma_{\dot{\delta}}$ denote the horizontal beam emittance and the rms momentum spread, respectively. The function $F(x)$ is defined as

$$
F(x)=\int_{0}^{1}\left(\frac{2}{u}-\ln \frac{1}{u}-2\right) \cdot \exp \left(-\frac{x}{u}\right) \mathrm{d} u .
$$

The Touschek lifetime depends linearly on both the bunch length $\sigma_{s}$ and the vertical beamsize $\sigma_{z}$, as Equation 1 indicates. However, the dependence on the horizontal beamsize (or the horizontal beam emittance) is more complicated. If the horizontal beamsize is large, the particle density within the bunch becomes very small, and subsequently the probability of two electrons coliding decreases. On the other hand, a large horizontal beam size corresponds to large horizontal angular divergence that can be transferred into the longitudinal direction due to a Touschek scattering event. This complicated dependence of the Touschek lifetime on the horizontal beam size is therefore best evaluated by numerical integration of Equation 1 for a fixed (positionindependent) value of the momentum acceptance $\delta_{\text {acc }}$. Figure 1 depicts the resulting Touschek lifetime in NSLS-II as a function of the horizontal beam emittance for a momentum acceptance of $\delta_{\text {acc }}=0.03$ and a vertical emittance of $1 \cdot 10^{-11} \mathrm{~m}$.

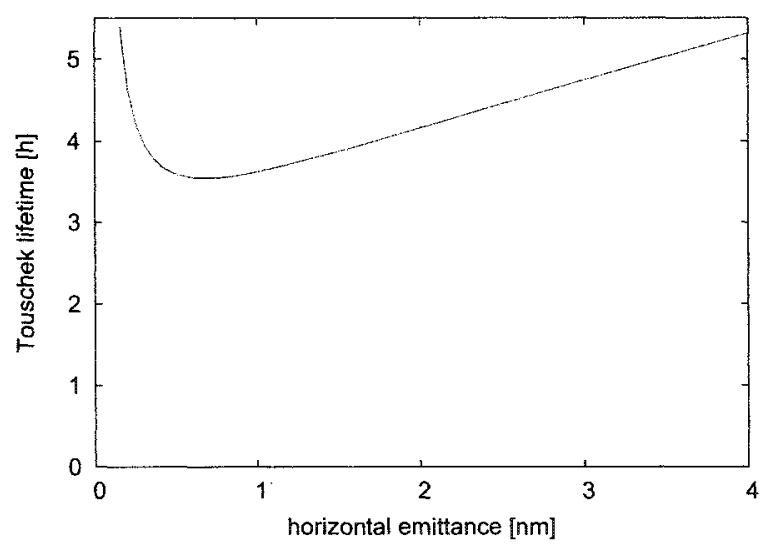

Figure 1: Touschek lifetime in NSLS-II as a function of horizontal emittance

\section{MOTIVATION}

While top-up injection relaxes the constraint on Touschek lifetime, it remains a limiting factor due to the increased demands on small beam emittance and current stability from the users. For a realistic design then, it is 
crucial that it is estimated correctly.

It is well known $[2,3,4,5,6]$ that e.g. the lattice symmetry and small ( $5 \mathrm{~mm}$ ) vertical apertures from mini-gap insertion devices have a strong impact on the Touschek life time. Losses can then occur due to coupling (linear and nonlinear) between the horizontal and vertical planes. Moreover, just because a particle is stable for some initial conditions in simulations without radiation damping, this does not mean that it is stable when damping is included. After a Touschek event, due to amplitude dependent tune shift and residual (linear and nonlinear) chromaticity, the particle crosses resonances and be lost during its return to equilibrium through damped (anharmonic) betatron oscillations. Correspondingly, to correctly estimate and control the Touschek life time, a realistic model is required. The model should also include the correction schemes for control of the dynamics for a realistic lattice. In other words, the dynamics for a nontrivial Hamiltonian needs to be evaluated, i.e., that includes: engineering tolerances, insertion devices, and corrections.

For a self consistent approach, the following aspects need to be included:

- magnetic alignment and field tolerances,

- damping wigglers and insertion devices,

- control of the impact of these on, i.e. orbit, vertical beam size [7], and the (linear) optics perturbations [8],

- physical apertures,

- and radiation damping.

Having established such a model, realistic estimates of the Touschek life time can then be provided. Guidelines can thus be worked out for e.g.:

- RF voltage (RF bucket).

- Top-up period.

- Min vertical physical aperture.

- Choice of working point.

- Can Touschek life time/momentum aperture be improved further?

- Lattice symmetry requirements?

- What are the beam loss patterns from top-up and Touschek scattering?

- Potential radiation damage of mini-gap undulators.

- Could vertical scrapers be used for effective control of the loss pattern; without significant reduction of the Touschek life time?

\section{A SELF CONSISTENT APPROACH}

We have implemented the ideas described in the previous section in order to get a realistic estimate of Touschek lifetime for NSLS-II. Misalignment errors with orbit correction have been applied to the design lattice. We also must add a realistic suite of damping wiggler and additional insertion devices for a realistic model. For these calculations, we have included three damping wigglers and one CPMU. Vertical emittance is controlled by adding vertical dispersion in such a way that coupling is not strongly increased $[9,7]$. The induced vertical dispersion wave is shown in Figure 2. The resulting vertical beam size is shown in Figure 3, and the coupling angle is shown in Figure 4. The vertical beam size should be set to the diffraction limited value. Larger than that degrades beam brithtness, whereas smaller than that degrades Touschek lifetime.

Once the lattice elements and physical apertures are appropriately set, the momentum aperture and resulting Touschek Lifetime is computed. Figure 5 shows the momentum aperture for the nominal ID gap width of $\pm 2.5 \mathrm{~mm}$. Note that in the dispersive regions, the aperture has decreased from its nominal value of $3 \%$.

Gap width is a crucial parameter to understand in relation to Touschek lifetime, and thus we have varied the gap in the CPMU and computed the resulting Touschek lifetime. Figure 6 shows a drop in lifetime in the 2 to $5 \mathrm{~mm}$ range. $2.5 \mathrm{~mm}$ has been chosen as a reference guideline for minimal gap size.

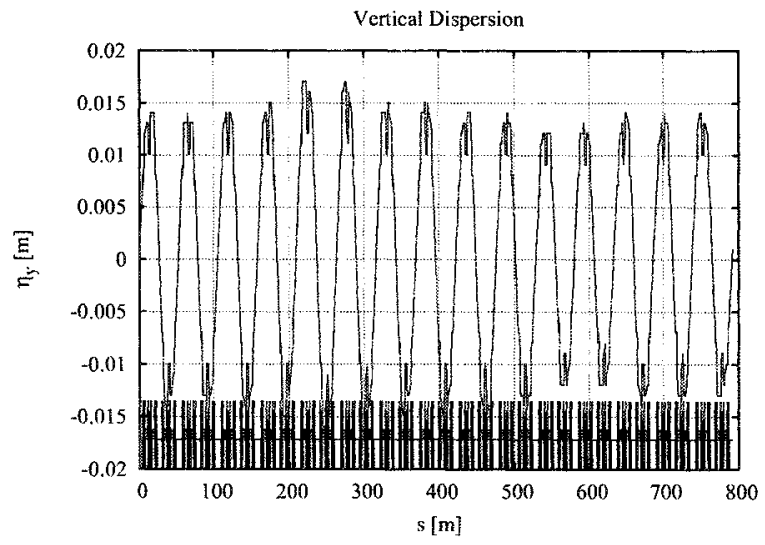

Figure 2: Vertical dispersion wave around the ring.

\section{CONCLUSIONS}

We have presented a summary of our approach to modelling Touschek lifetime for the NSLS-II. In particular, we have stressed the importance of self-consistent calculations in which the equilibrium beam sizes and the momentum aperture tracking both come from the same lattice model. Once the infrastructure for such calculations is in place, the results can be used to determine and optimize the Touschek lifetime. We have given one example of such optimization 


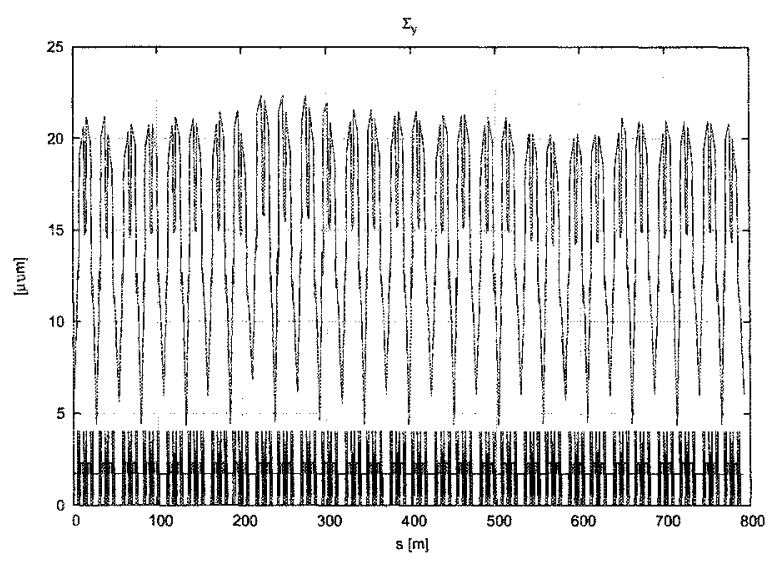

Figure 3: Vertical beam size around the ring.

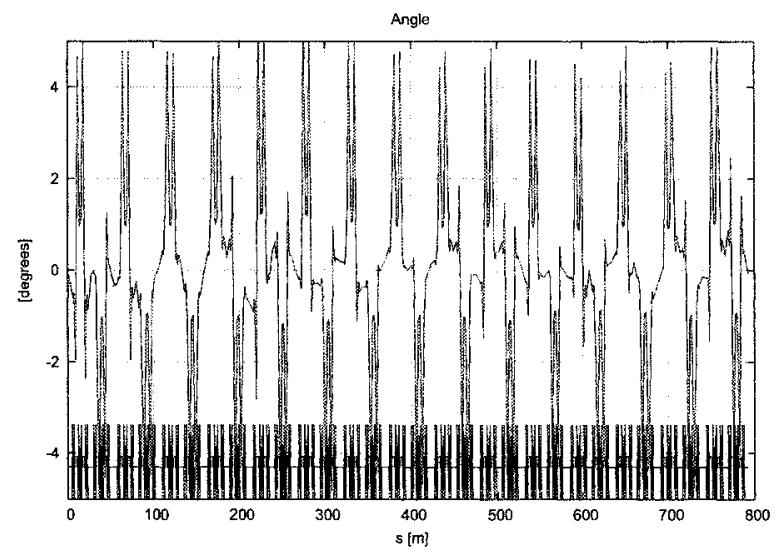

Figure 4: Transverse coupling angle around the ring.

by plotting the effect on lifetime of vertical gap size in the insertion devices. Because small vertical gap size and large Touschek lifetime are conflicting demands, such a detailed study is required in order to find an appropriate balance.

\section{ACKNOWLEDGMENTS}

Work performed under the auspices of the United States Department of Energy.

\section{REFERENCES}

[1] C. Bernardini et al., Phys. Rev. Lett. vol. 10 (1963) 407

[2] D. Robin, "Model Calibration and Symmetry Restoration of the Advanced Light Source", Proc. EPAC 1996

[3] A. Streun, "Momentum Acceptance and Touschek Lifetime", SLS-TME-TA-1997-0018 (1997)

[4] A. Streun, "Beam Lifetime of the SLS Storage Ring", SLSTME-TA-2001-0191 (2001)

[5] A. Nadji et al, "Refi ned Tracking Procedure for the SOEIL Energy Acceptance Calculation", Proc. PAC 2003

[6] C. Steier et al, "Measuring and Understanding the Dynamic Momentum Aperture in a Storage Ring", Proc. PAC 2005

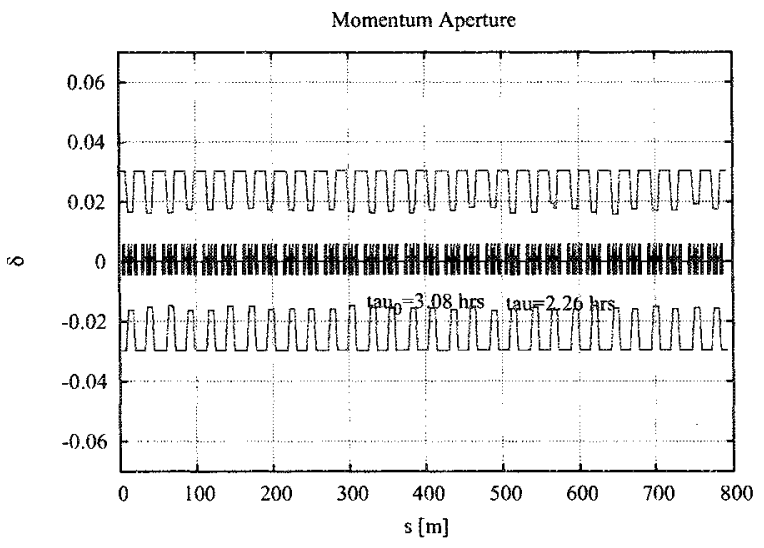

Figure 5: Momentum Aperture for vertical gap of $\pm 2.5 \mathrm{~mm}$ in CPMUs

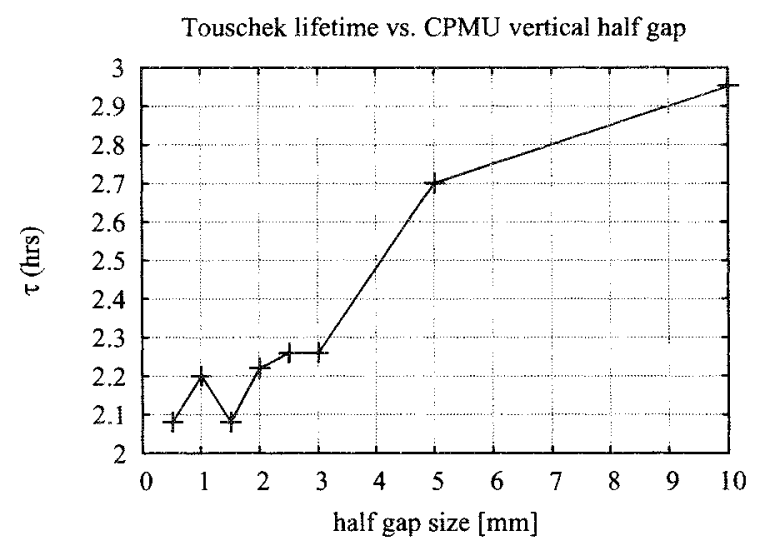

Figure 6: Touschek lifetime in NSLS-II for varying vertical gap size in CPMUs

[7] J. Bengtsson, I. Pinayev, "NSLS-II: Control of Vetical Beam Size", NSLS-II Tech Note 7 (2007)

[8] T. Shaftan et al, "Control of Dynamic Aperture with Insertion Devices", Proc. EPAC 2006

[9] C. Steier, "Coupling Correction and Beam Dynamics at Ultralow Vertical Emittance in the ALS", Proc. PAC 2003 